\title{
Is methylmercury a new candidate obesogen?
}

\author{
Gilles Tinant, Ineke Neefs, Jean-François Rees, Yvan Larondelle and Cathy Debier \\ UCLouvain, Louvain-la-Neuve, Belgium
}

\begin{abstract}
Recent studies suggest that environmental pollutants play a role in the continuous increase of the worldwide prevalence of obesity. To our knowledge, there is only one study investigating the impacts of methylmercury, a common and more toxic form of mercury, mainly present in fish and shellfish, on adipose tissue. It shows that methylmercury can cause changes in the structure and function of 3T3-L1 preadipocytes. In this context, we decided to study the effects of methylmercury on primary cultured adipocytes of rainbow trout. This species is a well-known model to study the impact of pollution. In addition, fish adipose tissue metabolism is close to the one of humans. Using an in vitro model allows us to focus on adipocyte differentiation and lipid metabolism.

Preadipocytes were obtained after isolating precursor cells from perivisceral adipose tissue by enzymatic digestion. Cells were cultured at $19^{\circ} \mathrm{C}$ until confluence before being incubated for 6 days in growth medium supplemented or not with a hormonal cocktail (insulin, dexamethasone, ciglitizone) enriched with $0,0.3,1.7$ or $3.8 \mathrm{mM}$ of methylmercury and lipid mixture. Cytotoxicity was evaluated every two days via LDH assay. At day 6, quantification of total mercury in cells was performed with Direct Mercury Analyser-80. Phospholipid and neutral lipid fractions were analyzed by gas chromatography and expression of adipocyte-related genes was evaluated by RT-PCR. Three biological replicates were carried out.

No cytotoxicity was observed during the methylmercury treatment. Methylmercury was partially accumulated but in a dosedependent manner. At day 6, all fatty acids (FA) from neutral lipid fraction significantly increased at the highest methylmercury concentration. However, the rate of increase was different for each FA. In particular, n-3 polyunsaturated FA were preferentially accumulated. The total amount of phospholipids was not impacted by methylmercury but a decrease of n- 6 polyunsaturated FA was observed. Surprisingly, methylmercury decreased the expression of three adipocyte markers, FA transport protein 1 and glycerol-3phosphate dehydrogenase in presence or absence of hormonal cocktail, and CCAAT/enhancer binding protein delta in presence of hormonal cocktail. On the other hand, methylmercury increased the expression of FA synthase and perilipin.

As a conclusion, methylmercury appears to be a candidate obesogen as it increased the cellular lipid content in primary cultured trout adipocytes. Additional genes involved in adipogenesis, FA de novo synthesis and FA intracellular transport are being investigated. Further experiments currently conducted in our lab should allow us to better clarify the mechanisms involved.
\end{abstract}

\section{Conflict of Interest}

There is no conflict of interest. 